INPLASY

PROTOCOL

To cite: Tan et al. Refraction shift after Nd: YAG posterior capsulotomy in pseudophakic eyes: a protocol for a systematic review and metaanalysis. Inplasy protocol 202120059. doi: 10.37766/inplasy2021.2.0059

Received: 17 February 2021

Published: 18 February 2021

Corresponding author: Yuan Tan

2016151621139@stu.scu.edu.cn

Author Affiliation:

Clinical Research Center, Zhongshan Ophthalmic Center, Sun Yat-sen University Guangzhou

Support: National Natural Science Found.

Review Stage at time of this submission: Preliminary searches.

Conflicts of interest:

None declared.

\section{Refraction shift after Nd: YAG posterior capsulotomy in pseudophakic eyes: a protocol for a systematic review and meta-analysis}

Tan, Y1; Li, W2 ; Liu, $Z^{3}$.

Review question / Objective: Nd: YAG laser treatment is widely used as an effective treatment modality for posterior capsule opacification(PCO). However, there is a lack of literature on refractive changes in pseudophakic eyes before and after Nd: YAG laser treatment. The results of various studies suggest that refractive changes after laser treatment are controversial. This study aims to perform a relatively credible and comprehensive assessment to compare the refractive changes that occur in pseudophakic eyes before and after laser treatment, as well as the positional changes, to suggest the need and timing of assessing the need for frame glasses or contact lenses for vision correction after Nd: YAG laser treatment.

Condition being studied: Laser treatment of PCO.

INPLASY registration number: This protocol was registered with the International Platform of Registered Systematic Review and Meta-Analysis Protocols (INPLASY) on 18 February 2021 and was last updated on 18 February 2021 (registration number INPLASY202120059).

\section{INTRODUCTION}

Review question / Objective: Nd: YAG laser treatment is widely used as an effective treatment modality for posterior capsule opacification(PCO). However, there is a lack of literature on refractive changes in pseudophakic eyes before and after $\mathrm{Nd:}$ YAG laser treatment. The results of various studies suggest that refractive changes after laser treatment are controversial. This study aims to perform a relatively credible and comprehensive assessment to compare the refractive changes that occur 
in pseudophakic eyes before and after laser treatment, as well as the positional changes, to suggest the need and timing of assessing the need for frame glasses or contact lenses for vision correction after Nd: YAG laser treatment.

Condition being studied: Laser treatment of PCO.

\section{METHODS}

Participant or population: Patients with PCO.

Intervention: Nd: YAG laser treatment.

Comparator: In the study with a control group, we will select the group of patients who had developed PCO without YAG laser treatment as a comparator. In the study without a control group, we will choose the patients' baseline status as a comparator.

Study designs to be included: We will consider all study types in which there is at least one study group in which laser treatment for PCO is used at any dose and for any treatment time in pseudophakic eyes.

Eligibility criteria: The study protocol will be developed and executed under Preferred Reporting Items for Systematic Reviews and Meta-analyses (PRISMA). The studies included in our meta-analysis will meet all of the following inclusion criteria: (1) Population: patients with PCO; (2) Intervention: Nd: YAG laser treatment; (3) Outcome: At least one of the following results: Spherical equivalent (SE), anterior chamber depth (ACD), or cylindrical error. Studies that meet any of the following criteria are about to be excluded: (1) Studies that did not compare the outcomes before and after laser treatment or set up a control group for the comparison; (2) Reviews or conference abstracts; (3) Case reports or letters. Two independent authors will screen titles and abstracts of potentially relevant studies to determine their eligibility based on the criteria. Disagreements will be resolved through discussions with a third author.
Information sources: The following databases will be searched: PubMed, EMBASE, Cochrane Database of Systematic Reviews, and the Cochrane Central Register of Controlled Trials. The bibliographies will also be searched for relevant literature.

Main outcome(s): Spherical equivalent (SE).

Additional outcome(s): Anterior chamber depth (ACD); cylindrical error.

Data management: The method of data extraction will follow that outlined in the Cochrane Handbook for Systematic Reviews of Interventions. The two independent authors will extract the following descriptive primary information from the selected studies: study characteristics such as authors, year of publication, study design, sample size, follow-up time, randomization, blinding, and outcome measures. The primary outcome is the Spherical equivalent. Secondary outcome measures are ACD and cylindrical error. Disagreements in data collection will be resolved through discussion with a third author. If data are missing or cannot be extracted directly, we will contact the appropriate author to ensure the information's integrity. Otherwise, data will be collected under the Cochrane Handbook for Systematic Reviews of Interventions. Extraction of incomplete data will be waived if necessary.

Quality assessment / Risk of bias analysis: The risk of bias in the quality assessment of the studies will be assessed by two independent researchers using the "Assessing the Risk of Bias of Individual Studies in Systematic Reviews of Health Care Interventions" scale for assessment. The checklist contains nine questions on selection, performance, attrition, detection, and reporting bias. The risk of bias for each study will be assessed independently by two authors. Disagreements will be discussed and resolved with a third author.

Strategy of data synthesis: The metaanalysis will be performed using Stata16 
based on the underlying characteristics of the included studies. When standard deviations of continuous variables at the end of the study are not reported, estimates are based on initial and final values, and correlations are obtained by calculation. Unless otherwise stated, random effects models will be used for all analyses. Heterogeneity will be estimated for each analysis, with the $I^{2}$ statistic used, as high heterogeneity suggests careful consideration of the results.

Subgroup analysis: If necessary, subgroup analyses will be performed on patient age, intraocular lens type, measurement tool, size of the laser-cut, energy of the laser, follow-up time, and quality of the literature.

Sensitivity analysis: When heterogeneity is significant, a sensitivity analysis will be conducted to identify potential sources of heterogeneity.

\section{Language: English.}

Country(ies) involved: China.

Keywords: Refraction shift; laser capsulotomy; intraocular lens; capsule opacification; meta-analysis.

Contributions of each author:

Author 1 - Yuan Tan designed the review and will write the initial manuscript.

Email: 2016151621139@stu.scu.edu.cn

Author 2 - Wei Li will conduct an initial search to determine feasibility, provide input into the study design, and review the manuscript.

Email: liwei329@mail2.sysu.edu.cn

Author 3 - Zhenzhen Liu conceived the topic and will lead the discussion when disagreements arise during literature screening and data collection, extraction, and analysis.

Email: liuzhenzhen@gzzoc.com 